\title{
Conuclear images of substructural logics
}

\author{
Giulia Frosoni \\ DIMA, Via Dodecaneso 35, 16146, Genova \\ frosoni@dima.unige.it
}

A famous result by McKinsey and Tarski ([5] and [6]) provides an interpretation of intuitionistic logic into the modal logic S4. The algebras for S4 are Boolean algebras with an interior operator. One wonders if it is possible to perform a similar construction starting from a logic which is different from classical logic, say a logic extending Full Lambek Calculus FL.

The algebraic counterpart of this construction is the so-called conuclear image of a variety of (pointed) residuated lattices. Recall from [3] that a conucleus on a residuated lattice $\mathbf{R}$ is a monotone map $\sigma$ from $R$ into $R$ satisfying the following identities: $\sigma(1)=1, \sigma(x) \leq x, \sigma(x) \leq \sigma(\sigma(x))$ and $\sigma(x) \cdot \sigma(y) \leq \sigma(x \cdot y)$ for all $x, y \in R$.

Since an interior operator on a Boolean algebra is a special example of conucleus, the following generalization of McKinsey and Tarski's interpretation comes naturally: given a substructural logic $\mathrm{L}$, denote by $\mathrm{L}_{\sigma}$ the logic $\mathrm{L}$ with an added unary operator $\sigma$ along with the axioms: ${ }^{1}$

(i) $(\sigma(A) \cdot \sigma(B)) \rightarrow \sigma(A \cdot B)$;

(ii) $\sigma(A) \rightarrow A$;

(iii) $\sigma(A) \rightarrow \sigma(\sigma(A))$;

and the necessitation rule $\frac{A}{\sigma(A)}$.

Then we can define an interpretation ${ }^{\sigma}$ of $\mathrm{L}$ into $\mathrm{L}_{\sigma}$ as follows: $p_{i}^{\sigma}=\sigma\left(p_{i}\right)$ $(i=1, \ldots, n, \ldots), 0^{\sigma}=\sigma(0), 1^{\sigma}=1,(A \cdot B)^{\sigma}=A^{\sigma} \cdot B^{\sigma},(A \vee B)^{\sigma}=A^{\sigma} \vee B^{\sigma}$, $(A \wedge B)^{\sigma}=\sigma\left(A^{\sigma} \wedge B^{\sigma}\right),(A \backslash B)^{\sigma}=\sigma\left(A^{\sigma} \backslash B^{\sigma}\right)$ and $(A / B)^{\sigma}=\sigma\left(A^{\sigma} / B^{\sigma}\right)$.

The conuclear image of $\mathrm{L}$ is the logic $\sigma(\mathrm{L})$ whose theorems are precisely those formulas $A$ of $\mathrm{L}$ such that $\mathrm{L}_{\sigma} \vdash A^{\sigma}$. Interestingly, $\sigma(\mathrm{L})$ is always a substructural logic (i.e., an extension of FL) and $\mathrm{L}$ is an extension of $\sigma(\mathrm{L})$. Moreover, if $\mathrm{L}$ is classical logic, $\sigma(\mathrm{L})$ is intuitionistic logic. Also, by a result proved in [7], the logic of commutative, cancellative residuated lattices is the conuclear image of the logic of lattice-ordered abelian groups.

The main problem we investigate in the paper is the following: what is the relationship between $\mathrm{L}$ and $\sigma(\mathrm{L})$ ? In particular:

(1) Which are the theorems of $\mathrm{L}$ which hold also in $\sigma(\mathrm{L})$ ?

(2) Which properties are excluded to hold in $\sigma(\mathrm{L})$, whatever $\mathrm{L}$ is?

(3) Which theorems are not necessarily preserved as in (1), nor excluded to hold as in (2)?

As regards to (1), we prove that all formulas corresponding to inequations of the form $f \leq g$ with $f \in P_{2}$ and $g \in N_{2}$ (where the classes $P_{2}$ and $N_{2}$, and more in general the classes $P_{n}$ and $N_{n}$, belong to the substructural hierarchy,

\footnotetext{
${ }^{1}$ When the distinction between $\alpha \backslash \beta$ and $\beta / \alpha$ is irrelevant, we denote either of them by $\alpha \rightarrow \beta$.
} 
see [2]) are preserved from $\mathrm{L}$ to $\sigma(\mathrm{L})$. Hence, every substructural logic which is axiomatized over FL by an inequation of this form coincides with its conuclear image.

Moreover, we generalize this result in such a way to include preservation of the cancellativity equation; to do this, we introduce new classes $P_{2}^{*}$ and $N_{2}^{*}$, which extend the corresponding classes of the substructural hierarchy, and provide a sufficient condition for the preservation under conuclear images. We also observe that if we relax this condition slightly, we find counterexamples of properties which are not preserved under conuclear images.

As regards to (3), interesting examples of axioms which are compatible with conuclear images but are not necessarily preserved are divisibility and distributivity. In order to prove this, observe that, while abelian lattice-ordered groups are distributive and divisible, there are commutative, cancellative residuated lattices which are not distributive or which are not divisible, see [1].

Finally, as regards to (2), we prove that the conuclear image of every substructural logic has the disjunction property. In view of this result, properties like excluded middle, weak excluded middle, prelinearity etc., never hold in a conuclear image. Moreover, by [4], the conuclear image $\sigma(\mathrm{L})$ of any substructural $\operatorname{logic} \mathrm{L}$, as well as its conuclear extension $\mathrm{L}_{\sigma}$, is PSPACE-hard.

There are also other properties, like the double negation axiom DN, which never hold in the conuclear image of any substructural logic. Since FL plus DN has the disjunction property ([8]), it follows that not all substructural logics with the disjunction property are conuclear images of a substructural logic. Being a conuclear image seems to be a stronger and more constructive property than the disjunction property.

It would be interesting to find a classification of axioms according to their constructive character and to show that constructive axioms are always preserved under conuclear images, that non-constructive axioms (like prelinearity, excluded middle, double negation) are never preserved, and that neutral axioms (like distributivity or divisibility) need not be necessarily preserved.

\section{References}

1. P.Bahls, J.Cole, N.Galatos, P.Jipsen, and C.Tsinakis, Cancellative residuated lattices, Algebra Universalis 50 (1), 83-106, (2003).

2. A.Ciabattoni, N.Galatos, and K.Terui, Algebraic proof theory for substructural logics: cut-elimination and completions, Annals of Pure and Applied Logic 163 (3), 266-290, (2012).

3. N.Galatos, P.Jipsen, T.Kowalski, and H.Ono, Residuated lattices: an algebraic glimpse at substructural logics, Studies in Logic and the Foundations of Mathematics, Volume 151, (Elsevier, Amsterdam, 2007), p.509.

4. R.Horčík and K.Terui, Disjunction property and complexity of substructural logics, Theoretical Computer Science 412 (31), 3992-4006, (2011).

5. J.C.C.McKinsey and A.Tarski, The algebra of topology, Annals of Mathematics 45 (1), 141-191, (1944).

6. J.C.C.McKinsey and A.Tarski, Some theorems about the sentential calculi of Lewis and Heyting, The Journal of Symbolic Logic 13, 1-15, (1948). 
7. F.Montagna and C.Tsinakis, Ordered groups with a conucleus, Journal of Pure and Applied Algebra 214 (1), 71-88, (2010).

8. D.Souma, An algebraic approach to the disjunction property of substructural logics, Notre Dame Journal of Formal Logic 48 (4), 489-495, (2007). 